\begin{tabular}{ll|l} 
Case Reports in & \multicolumn{2}{c}{ Case Rep Gastroenterol 2013;7:414-419 } \\
\cline { 2 - 3 } Gastroenterology & $\begin{array}{l}\text { DOI: 10.1159/000352015 } \\
\text { Published online: September 21, 2013 }\end{array}$ & $\begin{array}{l}\text { ○ 2013 S. Karger AG, Basel } \\
\text { www.karger.com/crg }\end{array}$ \\
\hline & $\begin{array}{l}\text { This is an Open Access article licensed under the terms of the Creative Commons } \\
\text { Attribution-NonCommercial 3.0 Unported license (CC BY-NC) (www.karger.com/OA- } \\
\text { license), applicable to the online version of the article only. Distribution permitted for non- } \\
\text { commercial purposes only. }\end{array}$
\end{tabular}

\title{
Unusual Images of Mass-Forming Intrahepatic Cholangiocarcinoma
}

\author{
Kazuki Takakura ${ }^{a}$ Mikio Kajihara ${ }^{a}$ Tetsuyoshi Iwasaki $^{a} \quad$ Daisuke Ide $^{a}$ \\ Tamihiro Miyazaki $^{a} \quad$ Yoshinori Arai $^{a} \quad$ Masayuki Saruta $^{a}$ Seiji Arihiro ${ }^{a}$ \\ Mika Matsuoka $^{a}$ Shigeo Koido ${ }^{a}$ Ryusuke Ito $^{b}$ Michinori Matsumoto $^{b}$ \\ Takeshi Gocho $^{\mathrm{b}}$ Shigeki Wakiyama ${ }^{\mathrm{b}}$ Katsuhiko Yanaga ${ }^{\mathrm{b}}$ Hisao Tajiria, \\ ${ }^{a}$ Division of Gastroenterology and Hepatology, Department of Internal Medicine, \\ ${ }^{b}$ Department of Surgery and ${ }^{\mathrm{C} D e p a r t m e n t}$ of Endoscopy, The Jikei University School of \\ Medicine, Tokyo, Japan
}

\section{Key Words}

Mass-forming intrahepatic cholangiocarcinoma - Moderately differentiated adenocarcinoma

\begin{abstract}
We experienced a case of mass-forming intrahepatic cholangiocarcinoma which could not been diagnosed accurately without pathologic findings. A 78-year-old Japanese woman with no particular symptoms was admitted for changes in liver function tests. Ultrasonography revealed a solid liver tumor. When there are no typical imaging features, no pathognomonic clinical findings and no obvious risk factors for any specific hepatic tumor, it may be difficult to make an accurate diagnosis before surgical resection. The lesion was resected on the basis of a high degree of suspicion for malignancy and submitted for pathologic evaluation. Microscopically, the neoplasm was a moderately differentiated adenocarcinoma with abundant fibrous stroma, consistent with a mass-forming cholangiocarcinoma. This case exemplifies the importance of considering the various tumorous and non-tumorous diseases in the differential diagnosis of a liver mass with atypical features, especially when malignancy cannot be excluded.

C 2013 S. Karger AG, Basel
\end{abstract}

\section{Introduction}

Intrahepatic cholangiocarcinoma (ICC) is a malignant tumor arising from epithelial cells lining the biliary tree and gallbladder [1], and is generally the second most prevalent intrahepatic primary cancer. It occurs in the middle-aged and elderly with no obvious sex 
Takakura et al.: Unusual Images of Mass-Forming Intrahepatic Cholangiocarcinoma

differences. Approximately 5-10\% of cholangiocarcinomas (CCs) are ICCs [2]. The rates of CC have been rising gradually worldwide over the past several decades. Generally, early stages of ICC do not produce specific clinical symptoms that are recognized by the affected persons, and there is no specific or practical laboratory method for the diagnosis in early stages. Hence, the resectability rate is low and diagnosis of tumors is frequently made when malignancies have progressed to an advanced stage beyond the limits of surgical treatment, with poor prognosis. The causes of this disease's lethality are not only its rapid growth, but also its tendency to invade adjacent organs and to metastasize. Curative resection is the most effective treatment and the only therapy associated with prolonged disease-free survival.

ICC, which is known to have the histological and molecular features of adenocarcinoma, can be classified into three types on the basis of gross morphologic features: mass-forming type, periductal infiltrating type and intraductal growth type. Of these, mass-forming CC is most common.

Since there are no specific blood tests that can diagnose CCs by themselves, ICCs are often diagnosed by imaging findings, and rarely during physical exams, as asymptomatic hepatic masses [3]. Not all tumors, however, show typical imaging findings, and they may mimic a variety of lesions such as hepatocellular carcinoma (HCC), liver metastases, biliary cystadenocarcinoma, mesenchymal tumor, parasitic liver disease and inflammatory pseudotumors; thus they are sometimes difficult to definitively diagnose preoperatively, despite the high resolution of the latest imaging techniques.

\section{Case Report}

A 78-year-old Japanese woman, with no particular physical indications like abdominal pain, generalized itching, weight loss, fever or jaundice, who was incidentally found to have changes in liver function tests, particularly an elevated level of alkaline phosphatase (368 U/l), was admitted to our hospital for investigation and treatment. She had no history of HBV or HCV infection or of excessive alcohol intake.

Ultrasonography revealed a solid liver tumor. Computed tomography also showed a large, heterogeneous and hypovascular tumor in the anterior segment; the tumor margin was smooth, clear and demonstrated ring enhancement and low density within the tumor both in early and delayed phase, suggesting degeneration, necrosis or a cystic lesion inside the tumor. Magnetic resonance imaging confirmed the presence of a mass lesion that was isointense on T1-weighted images and minimally hyperintense on T2-weighted images, with slightly lower intense fringe. The tumor was hyperintense on diffusion-weighted imaging. After the injection of intravenous contrast, the T1-weighted image showed peripheral ring enhancement on both early (fig. 1a) and delayed phases.

Enhanced magnetic resonance imaging showed that the tumor border was obvious; the tumor was isointense on T1-weighted imaging and showed slightly higher intensity than round on T2-weighted imaging and high intensity on diffusion-weighted imaging (fig. 1b).

Contrast-enhanced ultrasonography showed a tumor with isoechoic or mixed pattern; a dynamic study showed pycnomorphous tumor margin in the early phase, and the center of the tumor was heterogeneous and hypoechoic in the late phase. Defect re-perfusion imaging showed that the solid part of the tumor was hypervascular (fig. 1c). 3D volumetric analysis showed that the total liver volume was $1,041 \mathrm{ml}$ (right lobe $660 \mathrm{ml}$, left lobe $381 \mathrm{ml}$ ); the tumor volume was $24 \mathrm{ml}$ (fig. 2).

Laboratory testing showed normal liver enzymes, except for ALP (368 U/l): AST $18 \mathrm{U} / \mathrm{l}$, ALT $15 \mathrm{U} / \mathrm{l}$, total bilirubin $1.0 \mathrm{mg} / \mathrm{dl}$ and direct bilirubin $0.1 \mathrm{mg} / \mathrm{dl}$. Complete blood count 
Takakura et al.: Unusual Images of Mass-Forming Intrahepatic Cholangiocarcinoma

was normal (WBC 7,100/ $\mu \mathrm{l}$, RBC $4.30 \times 10^{6} / \mu \mathrm{l}$, PLT $343 \times 10^{3}$ and INR 1.0). Tumor markers were all within normal limits, e.g. CEA $4.2 \mathrm{ng} / \mathrm{ml}$, CA19-9 $14 \mathrm{U} / \mathrm{ml}$, AFP $3 \mathrm{ng} / \mathrm{ml}$, PIVKA-II $21.0 \mathrm{mAU} / \mathrm{ml}$, etc.

Although the laboratory and radiological findings were not typically pathognomonic for any specific hepatic tumor, the lesion was resected on the basis of a high degree of suspicion for malignancy and submitted for pathologic evaluation. Grossly, a $5.5 \times 4.0 \times 3.0 \mathrm{~cm}$ welldefined and non-encapsulated solid mass was identified (fig. 3a). Microscopically, the neoplasm was a moderately differentiated adenocarcinoma with abundant fibrous stroma, consistent with a mass-forming CC (fig. 3b). The patient's postoperative course was generally unremarkable.

\section{Discussion}

ICC is the second most prevalent liver cancer after HCC, accounting for $10-20 \%$ of all primary tumors of the liver [4]. Tumor appearance on the cut surface was categorized into three types according to the morphologic classification system proposed by the Liver Cancer Study Group of Japan: mass-forming (most common), periductal infiltrating, or intraductal growth type [5].

Mass-forming ICC is usually depicted as an irregular and homogeneous mass with low attenuation, minimal peripheral enhancement, focal dilatation of intrahepatic ducts around the tumor, and vascular encasement without the formation of a grossly visible tumor thrombus on computed tomography [6]. In this case, these diagnostic imaging characteristics were not seen. The magnetic resonance imaging features of mass-forming ICC are similar to those of its computed tomography imaging [7]. The mass shows an irregular margin with high signal intensity on T2-weighted imaging and low signal intensity on T1-weighted imaging [8].

Not all CCs manifest the same typical imaging patterns, and various atypical patterns are not unusual, as in this case. Mass-forming CC manifests as a homogeneous mass with an irregular but well-defined margin on ultrasonography. A peripheral hypoechoic rim is seen in about $35 \%$ of all tumors and consists of compressed liver parenchyma or proliferating tumor cells [9]. ICCs are difficult to distinguish from other solid intrahepatic masses as they lack specific ultrasonography features [10].

At gross pathologic examination, mass-forming ICC typically manifests as a homogeneous sclerotic mass with an irregular lobulated surface, usually without presence of hemorrhage or central necrosis [11]. The central portion of the tumor is composed of a variable degree of fibrosis and shows coagulative necrosis with scanty scattered tumor cells.

Several tumors or tumor-like lesions should be included in the differential diagnosis for mass-forming CC. HCC with cirrhotic stroma, sclerosing HCC and combined HCC-CC can all appear nearly identical to CC [12]. In addition, various tumors with abundant fibrous stroma, immature abscesses, metastasis from other sites, and hepatic tuberculosis may manifest similar findings.

Serum biochemical tests usually support the clinical suspicion of CC. Elevation of the serum levels of bilirubin and markers of biliary epithelial injury, such as alkaline phosphatase, are common. The most common tumor markers such as CA19-9 and CEA are also frequently used and often elevated in ICC, but they are not specific and are rarely diagnostic [13].

In most cases of ICC, there are several risk factors, like primary sclerosing cholangitis, chronic viral infection such as HBV and HCV, parasitic infection, intrahepatic biliary stones, 
Takakura et al.: Unusual Images of Mass-Forming Intrahepatic Cholangiocarcinoma

congenital biliary cystic diseases, liver cirrhosis, chemical agents, alcohol intake and smoking [14], which were all absent this case.

The only curative treatment for $\mathrm{CC}$ is surgical resection with negative margins. The use of liver volumetric and/or hepatic functional studies is warranted when anticipating an extended hepatic resection, to estimate the future liver remnant and minimize the risk of postoperative liver failure caused by insufficient function or small residual liver parenchyma. Our surgeons estimated by 3D volumetric analysis that there was indication of surgical resection in this case.

In summary, we experienced a case of unusual images of mass-forming ICC. There were no obvious risk factors for ICC. When there are no typical imaging features and no pathognomonic clinical findings, it may be difficult to make an accurate diagnosis, including the judgment of malignancy, before surgical resection. This case exemplifies the importance of considering the various tumorous and non-tumorous diseases in the differential diagnosis of a liver mass with atypical features, especially when malignancy cannot be excluded.

\section{Disclosure Statement}

No potential conflict of interest relevant to this article is reported.

\section{References}

1 Olnes MJ, Erlich R: A review and update on cholangiocarcinoma. Oncology 2004;66:167-179.

Patel T: Cholangiocarcinoma. Nat Clin Pract Gastroenterol Hepatol 2006;3:33-42.

Carriaga MT, Henson DE: Liver, gallbladder, extrahepatic bile ducts, and pancreas. Cancer 1995;75:171-190. Khan SA, Thomas HC, Davidson BR, Taylor-Robinson SD: Cholangiocarcinoma. Lancet 2005;366:1303-1314. Liver Cancer Study Group of Japan: Classification of Primary Liver Cancer. Tokyo, Kanehara, 1997, pp 6-8.

Lim JH: Cholangiocarcinoma: morphologic classification according to growth pattern and imaging findings. AJR Am J Roentgenol 2003;181:819-827.

7 Maetani Y, Itoh K, Watanabe C, et al: MR imaging of intrahepatic cholangiocarcinoma with pathologic correlation. AJR Am J Roentgenol 2001;176:1499-1507.

8 Choi BI, Lee JM, Han JK: Imaging of intrahepatic and hilar cholangiocarcinoma. Abdom Imaging 2004;29: 548-557.

9 Wernecke K, Henke L, Vassallo P, et al: Pathologic explanation for hypoechoic halo seen on sonograms of malignant liver tumors: an in vitro correlative study. AJR Am J Roentgenol 1992;159:1011-1016.

10 Bloom CM, Langer B, Wilson SR: Role of US in the detection, characterization, and staging of cholangiocarcinoma. Radiographics 1999;19:1199-1218.

11 Ros PR, Buck JL, Goodman ZD, Ros AM, Olmsted WW: Intrahepatic cholangiocarcinoma: radiologicpathologic correlation. Radiology 1988;167:689-693.

12 Fukukura Y, Hamanoue M, Fujiyoshi F, et al: Cholangiocellular carcinoma of the liver: CT and MR findings. J Comput Assist Tomogr 2000;24:809-812.

13 Chen CY, Shiesh SC, Tsao HC, Lin XZ: The assessment of biliary CA 125, CA 19-9 and CEA in diagnosing cholangiocarcinoma - the influence of sampling time and hepatolithiasis. Hepatogastroenterology 2002;49: 616-620.

14 Aljiffry M, Walsh MJ, Molinari M: Advances in diagnosis, treatment and palliation of cholangiocarcinoma: 1990-2009. World J Gastroenterol 2009;15:4240-4262. 


\section{Case Reports in \\ Gastroenterology}

\begin{tabular}{l|l}
\hline Case Rep Gastroenterol 2013;7:414-419 \\
\hline DOI: $10.1159 / 000352015$ & $\begin{array}{l}\text { C 2013 S. Karger AG, Basel } \\
\text { www.karger.com/crg }\end{array}$ \\
\hline
\end{tabular}

Takakura et al.: Unusual Images of Mass-Forming Intrahepatic Cholangiocarcinoma
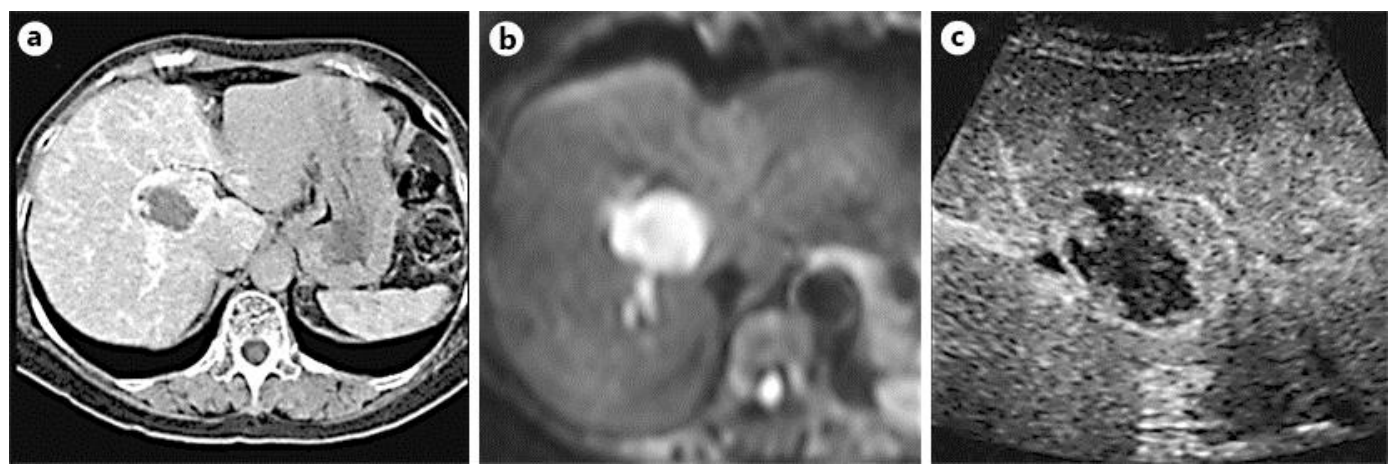

Fig. 1. a Findings of dynamic computed tomography of the abdomen (early phase). b Diffusion-weighed magnetic resonance imaging showed a markedly hyperintense signal. c Defect re-perfusion imaging of enhanced abdominal ultrasonography showed a hypervascular solid part of the tumor.

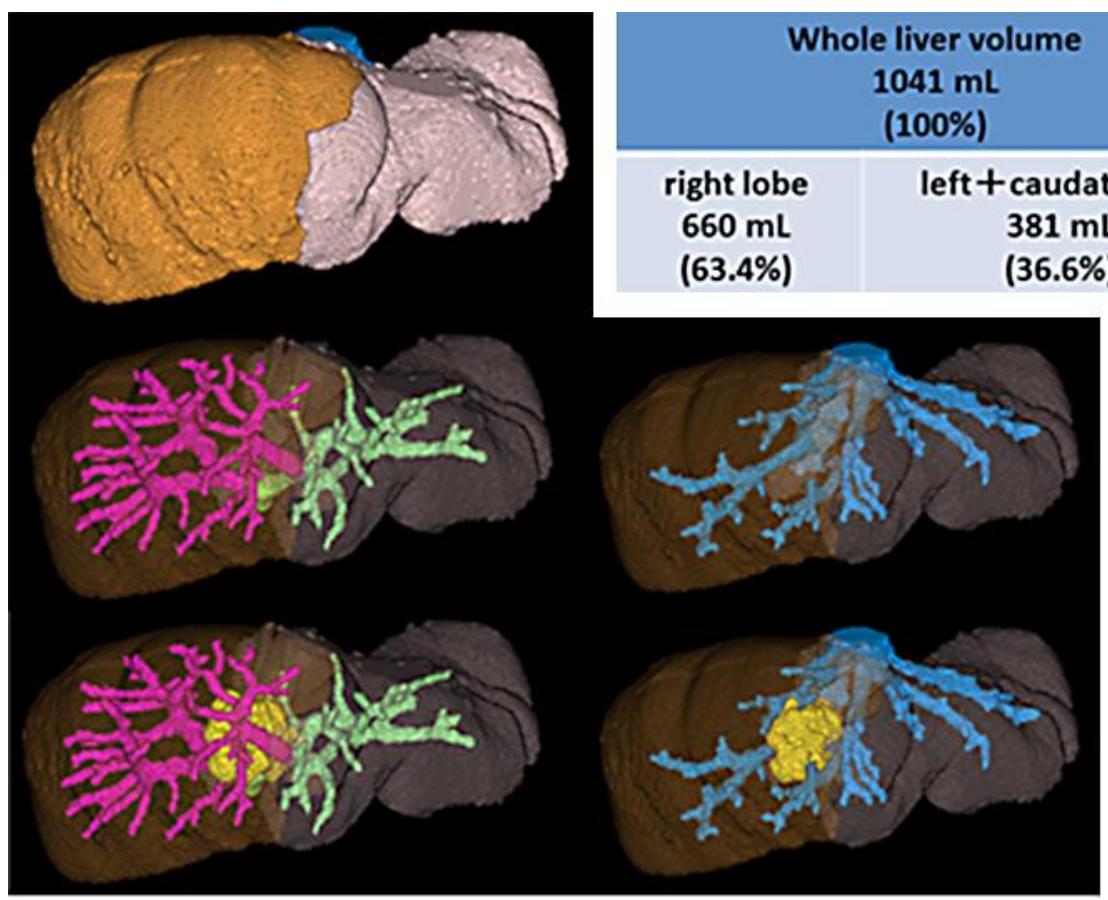

Fig. 2. 3D volumetric analysis showed the total volume of the liver and the tumor. 

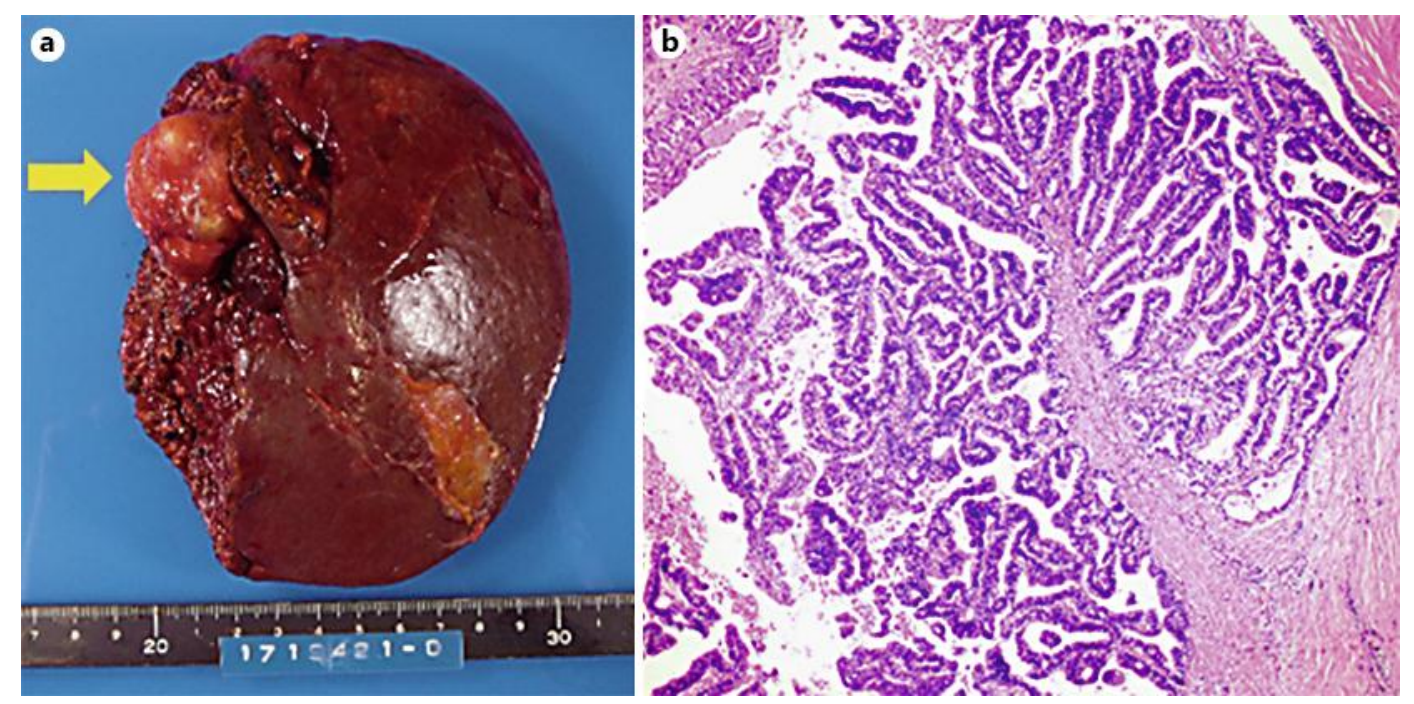

Fig. 3. a The photograph of the cut surface of the liver containing the tumor shows a yellowish-white, mass-forming solid tumor (arrow). The background liver is non-cirrhotic. b Pathologic findings. Photomicrograph (original magnification $\times 40$; hematoxylin-eosin stain) of the tumor periphery shows a moderately differentiated adenocarcinoma with marked periductal fibrosis. 